\title{
Fluid-Structure Interaction (FSI) of Damped Oil Conveying Pipeline System by Finite Element Method
}

\author{
Kah Soon Fong ${ }^{1, *}$ and Airil Yasreen Mohd Yassin ${ }^{1}$ \\ ${ }^{1}$ Department of Civil and Environmental Engineering, Universiti Teknologi PETRONAS, 32610 Seri Iskandar, Perak Darul Ridzuan, \\ Malaysia
}

\begin{abstract}
The vibration of damped pipeline conveying fluid with the effect of fluid-structure interaction is known to pose challenging problems in oil and gas industry. In this study, the natural frequency of fluidstructure interaction in pipeline conveying fluid set on viscoelastic foundation is investigated by using finite element method. The governing partial differential equation is modelled based on Euler-Bernoulli beam theory. By applying Galerkin weighted residual method, the stiffness, damping, and mass matrices are obtained. For a given boundary condition which is simply supported, two components of the foundation (foundation stiffness and damping) which are influencing the damped natural frequency of the pipeline are studied for different fluid velocity. The results indicate that increasing the foundation stiffness from $10 \mathrm{kN} / \mathrm{m}^{3}$ to $30 \mathrm{kN} / \mathrm{m}^{3}$ increases the natural frequency of the pipeline, while increasing the foundation damping from 1 $\mathrm{kN} . \mathrm{s} / \mathrm{m}^{3}$ to $3 \mathrm{kN} . \mathrm{s} / \mathrm{m}^{3}$ and fluid velocity decrease the natural frequency of the pipeline. The accuracy of the results obtained is validated against data from literature.
\end{abstract}

\section{Introduction}

Pipeline conveying fluid has a vital role in engineering fields particularly its application in marine engineering, oil and gas industry, oil transportation, and municipal water supply $[1,2]$. In oil and gas industry, pipelines are commonly used to convey and transport crude oil. However, the interaction between the fluid and structure often causes vibration problems to the pipelines, which has resulted in machinery breakdown, pipeline damages, fatigue failures, fires, and explosions in refineries and petrochemical plants. Consequently, huge economic loss due to pipeline vibration is experienced even by advanced country every year. The damage has been estimated as high as $\$ 10$ billion yearly [2]. Therefore, the research on the vibration characteristic of pipelines considering the effect of fluid-structure interaction such as natural frequency have gained the interest of researchers due to its great engineering and economic significance.

Pipeline conveying fluid is also considered as the simplest system of fluid-structure interaction problems, being able to describe significant dynamical behaviour of pipeline [3]. A century ago, the studies of pipeline conveying fluid were focused only on classic water hammer equation [4]. However, complex problems of pipeline conveying fluid were often encountered in various engineering fields. Therefore, it is necessary to consider the effect of fluid-structure interaction in the work due to its high development and demand in the field of engineering.

Important investigation in the study of vibration was made by Ashley and Haviland [5] which explained the vibration of the Trans-Arabian pipeline. The work involved a dynamic analysis of a simply supported pipe span using an approximate power series solution. However, the governing inertial force was not described adequately in the study as mentioned by Feodos'ev [6] Therefore, Feodos'ev formulated a more complete equation by considering the governing inertial force in the study. The equation was then solved by Galerkin method. Housner [7] developed a more realistic equation by considering the transverse vibration of the EulerBernoulli pipeline conveying fluid. The work presented several major factors such as elastic restoring force, centrifugal force, Coriolis force, and inertia force affect the vibration behavior of pipeline conveying fluid. Later, Paidoussis and Issid [8], Wiggert, et al. [9], Lesmez, et al. [10], and Wiggert [11] considered the transverse motion of the pipe in the equation of motion of pipeline. However, the equation did not contain any fluid related term except for Paidoussis and Issid work.

In recent years, a finite element formulation was developed by Sreejith, et al. [12] for a fully coupled dynamic equations of motions to study the liquid-filled pipeline's behaviour subjected to valve closure excitation. The study showed the significant reduction in structural velocities when the effect of fluid structure interaction was considered. Chellapilla and Simha [13] presented the vibration and stability characteristics of the pipeline conveying fluid based on the effect of two parameter foundation model. Critical velocity of the pipeline was obtained by using Fourier series and Galerkin method. A transverse vibration model was presented by Huang, et al. [1]. The dynamic equation with fluid structure interaction

\footnotetext{
* Corresponding author: kahsoon0401@gmail.com
} 
effect in the Euler-Bernoulli pipeline conveying fluid subjected to different boundary condition was solved using eliminated element-Galerkin method to obtain the natural frequency. The study showed that effect of the flow velocity on natural frequency can be estimated through its dimensionless fluid velocity and limit values. Another interesting finding from the study is that Coriolis effect can be neglected if the required precision of natural frequency is lower than 95. Huang, et al. [4] solved the same pipeline conveying fluid model for natural frequency analytically via separation of variables method and Ferrari's method..

In the present study, Galerkin finite element method is used to discretize the governing partial differential equation of one dimensional Euler Bernoulli pipeline conveying oil. The damped natural frequency of the pipeline is obtained through state space method with the imposed boundary condition of simply supported using the MATLAB program. The results are validated against the data reported by Mostafa [14].

\section{Governing equation of pipeline}

This study considers a horizontal pipeline rests on a viscoelastic medium that conveys oil as shown in Fig.1. The diameter of the pipeline is assumed to be comparatively smaller than the length of the pipeline. Therefore, the Euler-Bernoulli beam theory is applied to the pipeline model and the oil flow is approximated as plug flow model.

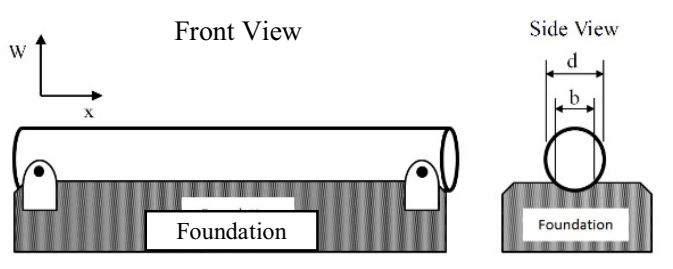

Pipe Conveying Fluid

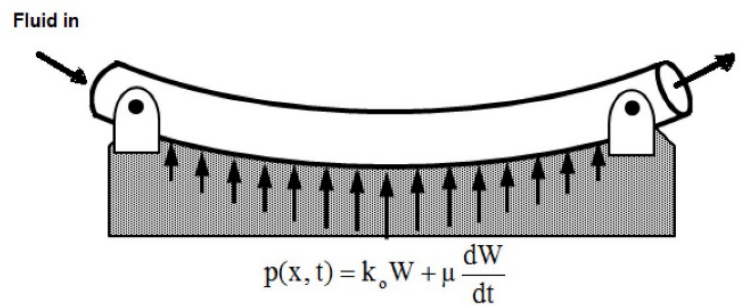

Fig. 1. Representation of modified Winkler foundation model[14]

The governing partial differential equation of motion that can represent the pipeline conveying oil on viscoelastic foundation based on the Euler-Bernoulli theory obtained from Mostafa [14] can be written as:

$$
E I \frac{\partial^{4} w}{\partial x^{4}}+k_{0} w+\mu \frac{\partial w}{\partial t}+M u^{2} \frac{\partial^{2} w}{\partial x^{2}}+2 M U \frac{\partial^{2} w}{\partial x \partial t}
$$

$$
+(m+M) \frac{\partial^{2} w}{\partial t^{2}}=0
$$

where $w(x, t)$ is the lateral pipeline displacement at position $x, E I$ is the bending stiffness of the pipeline, $u$ is the velocity of the fluid, $m$ is the pipe mass per unit length, $M$ is the conveying oil of mass per unit length, $k_{0}$ is the foundation stiffness coefficient per unit length and $\mu_{0}$ is the foundation damping coefficient per unit length.

The presence of Coriolis force is due to the relative motion of the fluid and is written as:

$$
F_{c}=2 M u(t) \frac{\partial^{2} w}{\partial x \partial t}
$$

The Coriolis force term $F_{c}$ is known as anti-symmetry whirligig "damping". Due to its effect, the finite element model becomes a complex eigenvalue problem.

\section{Finite element formulation}

The Galerkin weighted residual method is employed in the study as a tool for finite element formulation of pipeline governed by partial differential equation. The procedure is outlined in the following subsection.

\subsection{Shape function}

The guess solution of the lateral pipe displacement, $w(x, t)$ in an element can be expressed in term of Hermite cubic shape function as shown in Eq 3 or in form of component form as $\mathrm{Eq} 4$.

$$
\begin{gathered}
w(x, t)=N_{1} d_{1}+N_{2} d_{2}+N_{3} d_{3}+N_{4} d_{4} \\
w(x, t)=[N(x)]\{d(t)\}=N_{j} d_{j} \text { for } \mathrm{j}=1,2,3,4
\end{gathered}
$$

where $d_{1}$ and $d_{3}$ are nodal transverse degree of freedom while $d_{2}$ and $d_{4}$ are nodal rotational degree of freedom associated with node 1 and 2 respectively. The Hermite shape functions $N_{1}, N_{2}, N_{3}$, and $N_{4}$ are given as:

$$
\begin{gathered}
N_{1}=1-\frac{3 x^{2}}{L^{2}}+\frac{2 x^{3}}{L^{3}} \\
N_{2}=x-\frac{2 x^{2}}{L^{2}}+\frac{x^{3}}{L^{2}} \\
N_{3}=\frac{3 x^{2}}{L^{2}}-\frac{2 x^{3}}{L^{3}} \\
N_{4}=-\frac{x^{2}}{L}+\frac{x^{3}}{L^{2}}
\end{gathered}
$$

\subsection{Weak forms of differential equation}

Having established shape functions and degree of freedom of the beam element, the partial differential 
equation in Eq 1 can be discretized by substituting Eq 4 into it. Since Bubnov-Galerkin formulation is used in this paper, the weight functions used are the shape functions. Therefore, integrating the equation with the multiplication of weight function $N_{i}$ and it yields the equation given by:

$$
\begin{gathered}
\int_{0}^{l} N_{i}\left(E I \frac{\partial^{4}\left(N_{j} d_{j}\right)}{\partial x^{4}}+k_{0}\left(N_{j} d_{j}\right)+\mu \frac{\partial\left(N_{j} d_{j}\right)}{\partial t}\right) d x \\
+\int_{0}^{l} N_{i}\left(M u^{2} \frac{\partial^{2}\left(N_{j} d_{j}\right)}{\partial x^{2}}+2 M U \frac{\partial^{2}\left(N_{j} d_{j}\right)}{\partial x \partial t}\right) d x \\
+\int_{0}^{l} N_{i}\left((m+M) \frac{\partial^{2}\left(N_{j} d_{j}\right)}{\partial t^{2}}\right) d x=0
\end{gathered}
$$

By employing integration by part (IBP), the order of the original derivative terms is reduced. The final resulting weak form discrete finite element equation is written in the form of:

$$
\begin{gathered}
(m+M)\left[S_{1}\right]\{\ddot{d}\}+\left(\mu\left[S_{1}\right]+2 M U\left[S_{2}\right]\right)\{\dot{d}\} \\
+\left(E I\left[S_{3}\right]+\mathrm{k}_{0}\left[S_{1}\right]+M U^{2}\left[S_{4}\right]\right)\{d\}=0
\end{gathered}
$$

where,

$$
\begin{array}{ll}
{\left[S_{1}\right]=\int_{0}^{l}\left[N_{i}\right]\left[N_{j}\right],} & {\left[S_{2}\right]=\int_{0}^{l}\left[\frac{d N_{i}}{d x}\right]\left[N_{j}\right]} \\
{\left[S_{3}\right]=\int_{0}^{l}\left[\frac{d^{2} N_{i}}{d x^{2}}\right]\left[\frac{d^{2} N_{j}}{d x^{2}}\right],\left[S_{4}\right]=\int_{0}^{l}\left[\frac{d N_{i}}{d x}\right]\left[\frac{d N_{j}}{d x}\right]}
\end{array}
$$

\subsection{Finite element equations}

These equations can be rewritten in matrix form as finite element equation for each element:

$$
\left[m_{1}\right]\{\ddot{d}\}+\left(\left[\mathrm{c}_{1}\right]+\left[\mathrm{c}_{2}\right]\right)\{\dot{d}\}+\left(\left[k_{1}\right]+\left[k_{2}\right]-\left[k_{3}\right]\right)\{d\}=0(11)
$$

where $\left[m_{1}\right]$ is element mass matrix, $\left[c_{1}\right]$ and $\left[c_{2}\right]$ the element damping matrix, $\left[k_{1}\right],\left[k_{2}\right]$, and $\left[k_{3}\right]$ the element stiffness matrix, [4] the element displacement vector, $\{\dot{d}\}$ the element velocity vector, and $\{\ddot{d}\}$ the element acceleration vector. The respective element matrices are derived and shown below:

$$
m_{1}=\frac{(m+M) l}{420}\left[\begin{array}{cccc}
156 & 22 l & 54 & -13 l \\
22 l & 4 l^{2} & 13 L & -3 l^{2} \\
54 & 13 l & 156 & -22 l \\
-13 l & -3 l^{2} & -22 l & 4 l^{2}
\end{array}\right]
$$

$$
\begin{gathered}
c_{1}=\frac{\mu l}{420}\left[\begin{array}{cccc}
156 & 22 l & 54 & -13 l \\
22 l & 4 l^{2} & 13 l & -3 l^{2} \\
54 & 13 l & 156 & -22 l \\
-13 l & -3 l^{2} & -22 l & 4 l^{2}
\end{array}\right] \\
c_{2}=\frac{M U}{30}\left[\begin{array}{cccc}
-30 & -6 l & -30 & 6 l \\
6 l & 0 & -6 l & l^{2} \\
30 & 6 l & 30 & -6 l \\
-6 l & -l^{2} & 6 l & 0
\end{array}\right] \\
k_{1}=\frac{2 E I}{l^{3}}\left[\begin{array}{cccc}
6 & 3 l & -6 & 3 l \\
3 l & 2 l^{2} & -3 l & l^{2} \\
-6 & -3 l & 6 & -3 l \\
3 l & l^{2} & -3 l & 2 l^{2}
\end{array}\right] \\
k_{3}=\frac{M U^{2}}{30 l}\left[\begin{array}{cccc}
36 & 3 l & -36 & 3 l \\
3 l & 4 l^{2} & -3 l & -l^{2} \\
-36 & -3 l & 36 & -3 l \\
3 l & -l^{2} & -3 l & 4 l^{2}
\end{array}\right] \\
k_{o} l \\
\left.\begin{array}{cccc}
156 & 22 l & 54 & -13 l \\
22 l & 4 l^{2} & 13 l & -3 l^{2} \\
54 & 13 l & 156 & -22 l \\
-13 l & -3 l^{2} & -22 l & 4 l^{2}
\end{array}\right]
\end{gathered}
$$

\subsection{Dynamic eigenvalue of damped pipeline}

These local matrices are then assembled into global matrices to represent the full length pipeline model. After imposing boundary condition (simply supported), the matrices are arranged into proper form to determine the dynamic characteristic of the structure. The asymmetrical damping matric resulting from the Coriolis force cause the problem to be complex eigenvalue problem. In order to obtain the eigenvalues of damped structure, the standard equation of motion in the finite element form Eq 18 is converted to first order differential equation Eq 19 by introducing the state space vector $y_{1}=d$ and $y_{2}=\dot{d}$.

$$
\begin{gathered}
{[M]\{\ddot{d}\}+[C]\{\dot{d}\}+[K]\{d\}=0} \\
\dot{y}=\left[\begin{array}{l}
\dot{y}_{1} \\
\dot{y}_{2}
\end{array}\right]=\left[\begin{array}{cc}
0 & I \\
-M^{-1} K & -M^{-1} C
\end{array}\right]\left[\begin{array}{l}
y_{1} \\
y_{2}
\end{array}\right]
\end{gathered}
$$

By solving the first order differential equation, the natural frequency or eigenvalues and mode shapes or eigenvectors can be obtained. The solution of eigenvalue problem yields the complex roots and the imaginary part of the root represent the natural frequency of the damped structure as written in Eq 20. The real part of the root represent the rate of decay of the vibration.

$$
\lambda_{i}=-\xi_{i} \omega_{i} \pm \omega_{i} \sqrt{1-\xi_{i}^{2}} j
$$




\section{Numerical example}

To investigate the effectiveness and accuracy of present formulation, the results are compared with the numerical study reported [14] of a uniform simply supported pipeline. The natural frequency of the model is determined and compared by using the MATLAB program. The pipeline, which is $2 \mathrm{~m}$ long and made of steel with density $8000 \mathrm{~kg} / \mathrm{m}^{3}$, conveys the fluid with the density of $1000 \mathrm{~kg} / \mathrm{m}^{3}$. The thickness of the pipeline is of $0.001 \mathrm{~m}$ thick and has the outer diameter of $0.01 \mathrm{~m}$. The elastic modulus of pipeline is $207 \mathrm{GPa}$ and it is divided equally into 10 beam elements for the finite element analysis. The validated results using present formulation are shown and discussed in the following subsection.

\subsection{Effect of fluid velocity on natural frequency at different foundation stiffness}

This subsection investigates the natural frequency of a simply supported pipeline by varying the fluid velocity at different foundation stiffness. The present result is obtained by Galerkin finite element method while the result obtained by Mostafa [14] is based on energy finite element method. Both results are compared and excellent agreement between the results is achieved.

In Fig 2, the damped natural frequency of the pipeline with simply supported boundary condition for fluid velocity varying from $0 \mathrm{~m} / \mathrm{s}$ to $80 \mathrm{~m} / \mathrm{s}$ is illustrated. It is observed that the damped natural frequency decreases with an increase in fluid velocity. This is because higher fluid velocity weaken the structure's stiffness. In this example, the foundation viscous damping is set to be 100 $\mathrm{N} . \mathrm{s} / \mathrm{m}^{3}$ and the parameters of foundation stiffness are equalled to $10 \mathrm{kN} / \mathrm{m}^{3}, 20 \mathrm{kN} / \mathrm{m}^{3}$, and $30 \mathrm{kN} / \mathrm{m}^{3}$. The relation between the foundation stiffness and damped natural frequency is plotted in Fig. 2. The damped natural frequency increases with an increase of foundation stiffness because it increases its overall structure stiffness.

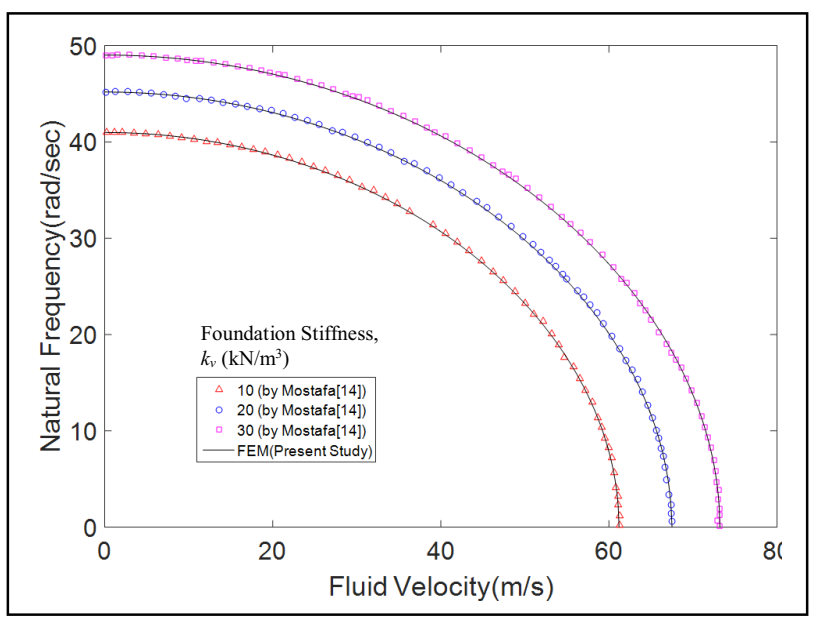

Fig. 2. Effect of fluid velocity on natural frequency at different foundation stiffness

\subsection{Effect of fluid velocity on natural frequency at different foundation viscous damping}

This subsection investigates the natural frequency of a simply supported pipeline by varying the fluid velocity at different foundation viscous damping. The present result is obtained by Galerkin finite element method while the result obtained by Mostafa [14] is based on energy finite element method. Both results are compared and excellent agreement between the results is achieved.

In Fig. 3, the damped natural frequency of the pipeline with simply supported boundary condition for fluid velocity varying from $0 \mathrm{~m} / \mathrm{s}$ to $80 \mathrm{~m} / \mathrm{s}$ is illustrated. Like previous discussion, the damped natural frequency decreases with an increase in fluid velocity. However, in this example, the parameter of foundation stiffness is set to be $20 \mathrm{kN} / \mathrm{m}^{3}$ and the foundation viscous damping are equalled to $1 \mathrm{kN} . \mathrm{s} / \mathrm{m}^{3}, 2 \mathrm{kN} . \mathrm{s} / \mathrm{m}^{3}$, and $3 \mathrm{kN} . \mathrm{s} / \mathrm{m}^{3}$. The relation between the foundation viscous damping and damped natural frequency is plotted in Fig. 3. Unlike previous result, the damped natural frequency decreases with an increase of foundation viscous damping. This is because the increment of foundation viscous damping leads to the reduction of dynamic properties of the pipe structure.

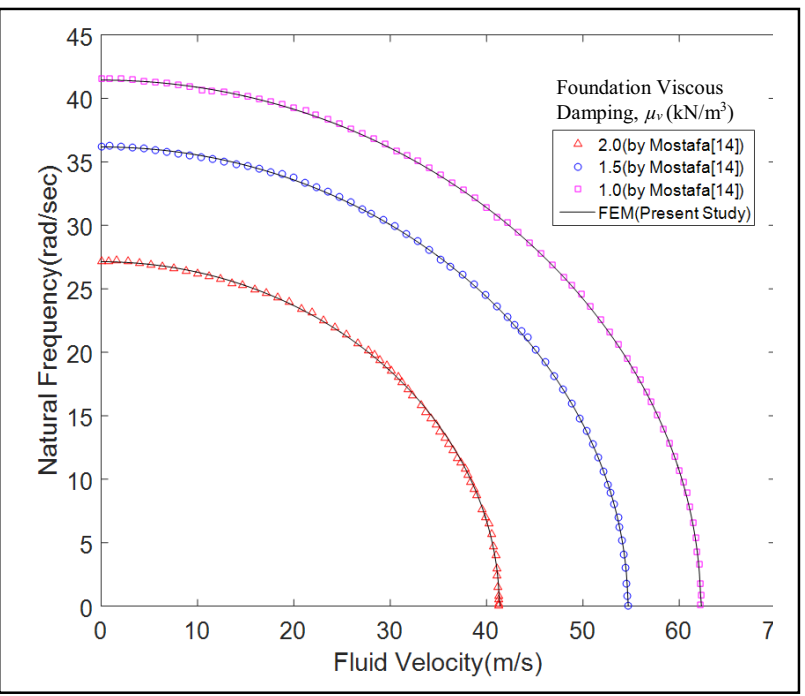

Fig. 3. Effect of fluid velocity on natural frequency at different foundation viscous damping

\section{Conclusion}

In this paper, the natural frequency of damped pipeline conveying oil is investigated and validated against the results of an existing work. Galerkin finite element method is employed to discretize the governing partial differential equation and the damped natural frequency of the structure are obtained though state space method. Several conclusions are drawn in this paper:

a) The presence of damping term, particularly external damping decreases the fluid critical velocity, while the increment of foundation stiffness increases the critical fluid velocity. 
b) For the eigenvalue problem, the inclusion of Coriolis force resulted in complex roots. The imaginary part of the roots indicates the natural frequency of damped structure.

c) Large centrifugal force resulting from high fluid velocity has weakened the stiffness of the structure and hence decreases the fluid critical velocity.

\section{References}

1. Y.-M. Huang, Y.-S. Liu, B.-H. Li, Y.-J. Li, and Z.-F. Yue, "Natural frequency analysis of fluid conveying pipeline with different boundary conditions," Nuclear Engineering and Design, vol. 240, pp. 461467, 2010.

2. Y. Sun and Y. G. a. H. Xiong, "Studied and Their Application of Vibration Control Technologies," 2013.

3. H. Dai, L. Wang, and Q. Ni, "Dynamics of a fluidconveying pipe composed of two different materials," International Journal of Engineering Science, vol. 73, pp. 67-76, 2013.

4. Y.-m. Huang, S. Ge, W. Wu, and J. He, "A direct method of natural frequency analysis on pipeline conveying fluid with both ends supported," Nuclear Engineering and Design, vol. 253, pp. 12-22, 2012.

5. H. Ashley and G. Haviland, "Bending vibrations of a pipe line containing flowing fluid," Journal of Applied Mechanics-Transactions of the ASME, vol. 17, pp. 229-232, 1950.

6. V. Feodos'ev, "Vibrations and stability of a pipe when liquid flows through it," Inzhenernyi Sbornik, vol. 10, pp. 169-170, 1951.

7. G. Housner, "Bending vibrations of a pipe line containing flowing fluid," Journal of Applied Mechanics-Transactions of the ASME, vol. 19, pp. 205-208, 1952.

8. M. P. Paidoussis and N. Issid, "Dynamic stability of pipes conveying fluid," Journal of sound and vibration, vol. 33, pp. 267-294, 1974.

9. D. Wiggert, F. Hatfield, and S. Stuckenbruck, "Analysis of liquid and structural transients in piping by the method of characteristics," Journal of fluids engineering, vol. 109, pp. 161-165, 1987.

10. M. W. Lesmez, D. Wiggert, and F. Hatfield, "Modal analysis of vibrations in liquid-filled piping systems," Journal of Fluids Engineering, vol. 112, pp. 311-318, 1990.

11. D. Wiggert, "Fluid transients in flexible piping systems," in Hydraulic Machinery and Cavitation, ed: Springer, 1996, pp. 58-67.

12. B. Sreejith, K. Jayaraj, N. Ganesan, C. Padmanabhan, P. Chellapandi, and P. Selvaraj, "Finite element analysis of fluid-structure interaction in pipeline systems," Nuclear Engineering and Design, vol. 227, pp. 313-322, 2004.

13. K. R. Chellapilla and H. Simha, "Critical velocity of fluid-conveying pipes resting on two-parameter foundation," Journal of sound and vibration, vol. 302, pp. 387-397, 2007.

14. N. H. Mostafa, "Effect of a Viscoelastic Foundation on the Dynamic Stability of a Fluid Conveying Pipe,"
International Journal of Applied Science and Engineering 12 (1), pp. 59-74, 2014. 